\title{
Estrutura e dinâmica em redes interorganizacionais: estudo de caso da rede de
}

\section{inovação da Fiocruz Paraná}

Leandro Rodrigo Canto Bonfim

Doutorando em Administração da Escola de Administração, Programa de Pós-Graduação em Administração (PPGADM) da Universidade Federal do Paraná. Pesquisador visitante (doutoradosanduíche) no departamento Management \& Organization da The Pennsylvania State University (Penn State), Estados Unidos.

lrcbonfim@ufpr.br

Sandro Aparecido Gonçalves

Pesquisador do Instituto Brasileiro de Estudos e Pesquisas Sociais (IBEPES). Doutor em Administração de Empresas pela EAESP/FGV

sandro1313@uol.com.br

Andréa Paula Segatto

Professora Associada da Escola de Administração, Coordenadora do Programa de Pós-Graduação em Administração (PPGADM) da Universidade Federal do Paraná (UFPR). Doutora em Administração pela Universidade de São Paulo (USP)

andreapsegatto@gmail.com

\begin{tabular}{|c|} 
Editor Científico: José Edson Lara \\
Organização Comitê Científico \\
Double Blind Review pelo SEER/OJS \\
Recebido em 28.06.2018 \\
Aprovado em 18.08.2018
\end{tabular}




\title{
Resumo
}

A dinâmicas das redes interorganizacionais têm se tornado tema de interesse nos estudos da área de negócios. Assim, a presente investigação buscou analisar como se deu a formação e a evolução de uma rede de inovação em biologia molecular biologia molecular voltada para os diagnósticos em Saúde Pública no sul do Brasil por meio de uma organização focal. O método adotado foi a análise de redes sociais (ARS) com recorte temporal transversal com aproximação longitudinal, a partir de evidências documentais fornecidas pela organização e de dados secundários. Os resultados demonstraram que a dinâmica da rede favoreceu a sua abertura, com o surgimento de buracos estruturais, porém, com adensamento das relações de proximidade e confiança com os laços vizinhos. Ademais, percebeu-se maior abertura da rede quanto aos laços com organizações de origem e finalidades distintas. Este estudo contribuiu ao ampliar a compreensão acerca da formação e evolução de redes de inovação.

Palavras-chave: Redes interorganizacionais. Dinâmica de redes. Inovação. Biotecnologia. Análise de redes sociais.

\section{Structure and dynamics in interorganizational networks: case study of the Fiocruz Paraná innovation network}

\begin{abstract}
Network dynamics has been a growing topic of interest in the business field. Thus, this study aimed to analyze how an innovation network in molecular biology applied to diagnostics in public health was formed and evolved. The method adopted were the cross-section social network analysis (SNA), with a longitudinal approximation. The source of evidence were documentary data provided by the hub organization and secondary data. The results showed that the network dynamics led to its openness, with the emergence of structural holes, but maintaining the density in close relationships with neighbor ties. Furthermore, it was perceived an openness regarding ties with organizations of distinct origin and kinds. This study contributed by extending the comprehension about the formation and evolution of innovation networks.
\end{abstract}

Keywords: Interorganizational networks. Network dynamics. Innovation. Biotechnology. Social network analysis.

Estructura y dinámica en redes interorganizacionales: estudio de caso de la red de innovación de Fiocruz Paraná

\section{Resumen}

Las dinámicas de las redes interorganizacionales se han convertido en un tema de interés del área de negocios. Así, la presente investigación buscó analizar cómo se dio la formación y la evolución de una red de innovación en biología molecular orientada hacia los diagnósticos en Salud Pública en el sur de Brasil a través de una organización focal. Se adoptó el análisis de redes sociales (ARS) con recorte temporal transversal con aproximación longitudinal, a partir 
de evidencias documentales y datos secundarios. Los resultados demostraron que la dinámica de la red favoreció su apertura, con el surgimiento de agujeros estructurales, sin embargo, con el adensamiento de las relaciones de proximidad y confianza con los lazos vecinos. Se percibió mayor apertura de la red en cuanto a los vínculos con organizaciones de origen y finalidades distintas. Este estudio contribuyó a ampliar la comprensión de la formación y evolución de redes de innovación.

Palabras clave: Redes interorganizacionales. Dinámica de redes. Innovación. Biotecnologia. Análisis de redes sociales.

\section{Introdução}

Durante muitos anos os estudos de redes sociais têm se caracterizado pelas relações estáticas, de modo que tal tendência à estabilidade pode ser revelada pela dominância dos estudos transversais (Burt, 2000). Deste modo, há uma primazia de se ver apenas a foto, e não o filme das relações, sejam elas intraorganizacionais ou interorganizacionais. Ao analisar a dinâmica das redes por meio da sua formação e evolução, pauta-se no princípio de que com o passar do tempo os laços se modificam. Nesse sentido, relações enfraquecem e se dissolvem, outras se tornam mais fortes e confiáveis, assim como novos laços são formados. Assim, "as memórias ocultas dos laços passados podem diluir ou modificar os efeitos das estruturas de rede antigas no comportamento e no desempenho presente" (Ahuja, Soda, \& Zaheer, 2012, p. 441).

Entretanto, tem se buscado compreender as dinâmicas entre os fatores institucionais e relacionais, conectando os estudos das redes com os campos organizacionais em uma perspectiva temporal longitudinal (Ahuja et al., 2012; Powell, White, Koput, \& Owen-Smith, 2005; Soda, Usai, \& Zaheer, 2004; Vasudeva, Zaheer, \& Hernandez, 2013). Deste modo, análise estrutural das dinâmicas de redes recentemente têm se mostrado prolíficas nos estudos de desempenho (Soda et al., 2004), inovação e inovatividade (Ahuja, 2000; Vasudeva et al., 2013), pesquisa e desenvolvimento (Amburgey, Al-Laham, Tzabbar, \& Aharonson, 2008), mudanças tecnológicas (Funk \& Owen-Smith, 2017), e comunicação organizacional (Lee \& Monge, 2011).

Tendo em vista a necessidade de se entender não apenas como os laços são formados e desfeitos, mas como os campos são formados e estruturados por meio de tais dinâmicas, o presente artigo teve como objetivo analisar como se deu a formação e a evolução de uma rede de inovação em biologia molecular voltada para os diagnósticos em Saúde Pública no Paraná. O estudo contribuiu ao demonstrar como a rede se configurou estruturalmente em termos de 
abertura e fechamento da rede, relações entre organizações distintas (origem, finalidade, caráter público ou privado), confiança, fluxo de conhecimento, e a memória da rede, proporcionando que ocorressem inovações e desenvolvimento tecnológico.

O artigo está estruturado da seguinte maneira. Primeiro, é apresentado referencial teórico acerca de redes interorganizacionais e a imersão relacional e estrutural das redes. Apresentamse também os procedimentos metodológicos adotados na pesquisa. A seguir, realiza-se a análise dos dados em dois momentos no tempo, entre 1999 e 2007, e entre 2008 e 2014. Por fim, são discutidos os resultados, apresentadas as limitações e possibilidades futuras de pesquisa.

\section{Redes interorganizacionais}

Apesar de a análise de redes já ser promissora nas ciências sociais desde a década de 1970 (Emirbayer \& Goodwin, 1994), a análise de redes interorganizacionais começou a ganhar destaque somente a partir de meados da década de 1990 devido em grande parte à criação e consolidação da União Europeia (Grandori, 1997), de modo que a organização em forma de redes interorganizacionais tornou-se desde então "cada vez mais importante na vida econômica" (Grandori \& Soda, 1995, p. 183), assumindo papel de destaque especialmente nos estudos em estratégia organizacional (Gulati, Nohria, \& Zaheer, 2000; Phelps, 2010; Rowley, Behrens, \& Krackhardt, 2000).

Junto com o destaque dado à análise das redes interorganizacionais vieram discussões conceituais. Uma das discussões recorrentes em microeconomia e consequentemente em estratégia é o dilema entre produzir (e incorrer em custos de produção e burocracia) e comprar (e incorrer em custos de transação), ou seja, o dualismo entre mercado e hierarquia (Granovetter, 1985; Thorelli, 1986). A discussão ganha contornos mais conflitantes quando surge o problema das redes. Duas posições distintas são assumidas: (a) as redes são vistas como uma estrutura de governança ou arranjo produtivo híbrido entre mercado e hierarquia, sendo o melhor dos mundos entre ambos (Thorelli, 1986; Williamson, 1991; Wood Jr., 2010); (b) as redes não são consideradas nem mercado e nem hierarquia, mas "uma forma distinta de coordenar a atividade econômica" (Powell, 1990, p. 301), ou seja, uma terceira via com características tão peculiares que não podem ser tratadas como um meio termo entre os outros dois tipos de arranjos produtivos. 
Para defender a posição das redes como uma terceira forma de organização, Powell (1990) elaborou um modelo no qual comparou a estrutura de mercado, de hierarquia e de redes. Ao analisar o modelo do autor, as diferenças entre as formas de organização ficam evidentes, sendo que as redes possuem características bem peculiares que podem conferir às organizações algumas vantagens e desvantagens. Em relação ao mercado, vantagens como a redução dos custos de transação e a facilidade de acesso a recursos que a organização não teria isolada (Powell, Koput, \& Smith-Doerr, 1996) podem ser contrapostas pelo risco de oportunismo e quebra de confiança e pela menor flexibilidade na tomada de suas decisões (Abosag, Yen, \& Barnes, 2016). Já em relação à hierarquia, as redes têm as vantagens de redução dos custos de burocracia e o aumento da capacidade de aprendizagem por meio dos demais parceiros da rede, entretanto também possuem desvantagens, como a interdependência das escolhas e das decisões, resultando na falta de autonomia administrativa (Powell, 1990).

Lavie (2006) aponta, contudo, que a estruturação da rede pode diferir de acordo com a homogeneidade de recursos, sendo possíveis duas maneiras de organização: (a) a organização pode estar em redes com conjuntos de recursos homogêneos, na qual a organização focal possui sua capacidade de lucratividade aumentada "baseada na experiência acumulada dos parceiros similares, mecanismos de governança mais eficientes e barganha ampliada pela redução da dependência de cada parceiro" (Lavie, 2006, p. 650); e (b) a organização pode estar organizada em redes com recursos heterogêneos, na qual recursos complementares reduzem os riscos e os custos tecnológicos, possibilitam o crescimento e potencializam a inovação.

\section{Imersão relacional e estrutural de redes}

Em relação aos critérios desejáveis de imersão estrutural, Burt (2001) aponta que duas posições opostas são defendidas (e que refletem também duas posições antagônicas em relação à imersão relacional). A primeira, fundada por Coleman (1988), defende que a imersão estrutural ótima para as organizações em rede é densa ou fechada. Nessa configuração, uma rede formada pelos atores $\mathrm{A}, \mathrm{B}, \mathrm{C}, \mathrm{D}$, todos os nós (cada nó representa um ego da rede) estão ligados entre si (ver Figura 1a). 


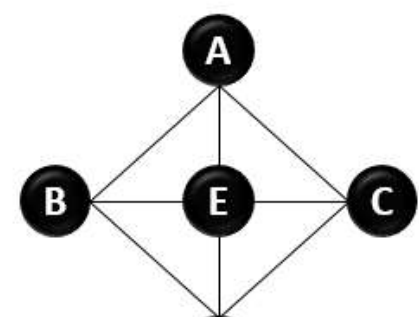

$1 \mathrm{~A}$

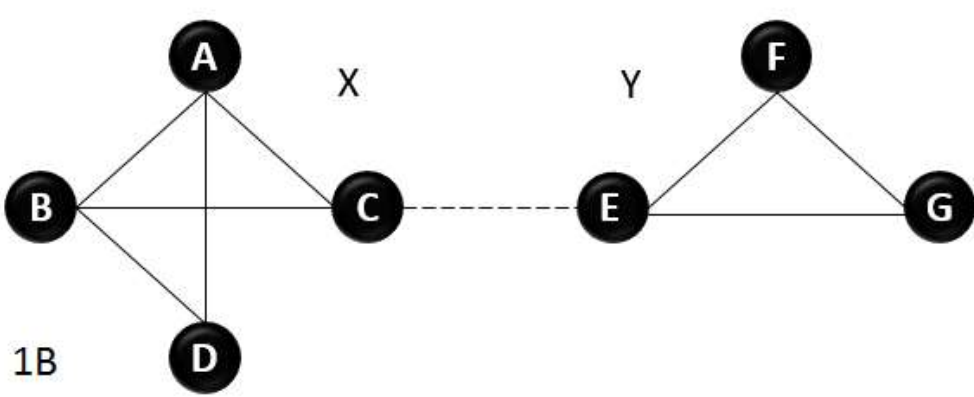

Figura 1. Representação gráfica de rede densas ou fechadas (1a) e redes esparsas e com buracos estruturais (1b)

As vantagens conferidas a esse tipo de organização em rede são a maior confiabilidade entre os membros da rede, maior possibilidade de sanções em caso de desvios de conduta, melhor fluxo de informações e a maior probabilidade de as informações serem confiáveis. A imersão relacional que melhor se enquadra com as definições de Coleman (1988) é a dos laços fortes. Um dos mais respeitados teóricos de redes e defensor dos laços fortes, Krackhardt (1992) argumenta que os laços fortes são capazes de garantir estabilidade em momentos de incerteza, além de serem bases de confiança nas relações.

A segunda posição de imersão estrutural foi fundada pelos estudos de Burt (1992). O autor argumentou que não é a forma de organização em redes densas (fechadas) a melhor configuração para o desempenho da rede. Para esta vertente, é fundamental que as redes possuam estruturas esparsas, possuindo o que ele chamou de buracos estruturais. Os buracos estruturais são as "relações de não redundância entre os contatos" (Burt, 1992, p. 65), de modo que tais relações são facilitadoras da inovação, visto que otimizam o fluxo de informações que ainda não estão disponíveis na rede.

Nessa forma de organização, atores que funcionam como pontes trazem as informações de fora para dentro da rede, conectando a rede $\mathrm{X}$ com a rede $\mathrm{Y}$ sem a necessidade que todos os atores pertencentes a cada rede estejam ligados entre si (ver Figura 1b, os nós A, B e D têm acesso aos nós $\mathrm{E}, \mathrm{F}$ e $\mathrm{G}$ somente por meio da ponte realizada por meio de $\mathrm{C}$, do mesmo modo, D funciona como ponte entre os nós da rede $\mathrm{Y}$ e os nós da rede $\mathrm{X}$, ou seja, C e D são buracos estruturais em suas redes). A imersão relacional ligada à ideia de buracos estruturais é a de laços fracos de Granovetter (1973), visto que o autor afirma que somente os laços fracos podem servir de pontes entre redes distintas.

A forma estrutural da rede, depende de diversas variáveis, sendo determinantes, como afirmado acima, o contexto e a finalidade da rede. Existem diversos tipos de redes 
interorganizacionais organizadas de diversas maneiras diferentes (Powell et al., 1996), havendo redes mais simples que integram as relações verticais entre os componentes de uma cadeia de valor (e.g. polos automobilísticos) até redes mais complexas, as quais chegam a envolver elevados níveis de trocas de tecnologia e de integração. Este segundo tipo é particularmente encontrado em redes em contexto hiperdinâmico com foco em produtos e serviços que demandam altos níveis de inovação tecnológica, como no caso da formação e crescimento dos startups do Vale do Silício (Castilla, Hwang, Granovetter, \& Granovetter, 2000).

\section{Dados e métodos da pesquisa}

O objetivo deste artigo foi analisar como se deu a formação e a evolução dessa rede de inovação em biologia molecular voltada para os diagnósticos em Saúde Pública. Para isso, adotou-se uma estratégia de pesquisa com análise de redes sociais (ARS) com recorte temporal transversal com aproximação longitudinal. A principal fonte de evidência foram os dados documentais fornecidos pela Fiocruz Paraná, dentre os quais encontravam-se projetos, atas de reuniões, estatutos de organização, e dados disponibilizados em websites.

A análise da rede foi desenvolvida no software Ucinet VI (Borgatti, Everett, \& Freeman, 2002), de modo que os dados foram organizados em matrizes sociométricas para cada período estudado e que deram origem aos sociogramas apresentados. Para melhor compreensão dos dados, as matrizes sociométricas foram construídas valoradas, possibilitando gerar a visualização da rede com a força dos laços entre os atores, assim como visualizar a distribuição de centro-periferia pela centralidade de grau. A força dos laços foi mensurada pela frequência das relações dispostas em matrizes sociométricas simétricas e de adjacência. Deste modo, quanto maior a frequência de interação entre dois nós, ou seja, a quantidade de projetos compartilhados entre os atores, maior a força do laço.

Para extração do coeficiente de agrupamento, assim como dos buracos estruturais, e de outras medidas extraídas ao longo da análise, foi utilizada a matriz dicotômica, ou binária, de modo que a mesma reduz a incidência de inconsistências nos cálculos, visto que o código do UCInet V® (Borgatti et al., 2002) opera de modo mais eficiente com matrizes dicotômicas (Hanneman \& Riddle, 2005). Pondera-se que para essas medidas a força dos laços expressa pela frequência das relações não é relevante ao seu cálculo. O tamanho efetivo, ou effective size, é uma das medidas de buracos estruturais mais adotadas, e seu cálculo consiste em verificar a redundância das relações, de modo que a fórmula expressa por Burt (1992) é determinada pelo 
número de alteregos que um ego possui menos a média de laços que cada alterego possui com outros alteregos (Hanneman \& Riddle, 2005).

Para avaliar a partição da rede em subgrupos, adotou-se a técnica de conjuntos Lambda. Ao identificar os pontos de vulnerabilidade da rede, buscam-se as pontes que, caso removidas, desconectariam a rede como um todo (Hanneman \& Riddle, 2005). Borgatti, Everett e Shirey (1990) apontaram que quanto maior o valor de Lambda $(\lambda)$, mais difícil fica de desconectar o subgrupo ao se remover nós limítrofes e menos vulnerável o grupo é à dirupção.

Para realizar essa análise das as relações entre os grupos e dentro dos grupos, foram criadas matrizes de afiliação (redes 2-mode) para cada rede com os seguintes atributos: origem da organização (por país, visto que houve colaboração com membros de outros países), por caráter (público ou privado) e por finalidade (universidade, instituto de pesquisa, indústria, associação, instituto técnico, laboratório biológico ou químico, hospital ou órgão governamental de controle). Uma quarta categoria foi criada para analisar a endogenia dessas relações entre unidades da Fiocruz em específico. A técnica adotada para análise foi desenvolvida por Krackhardt e Stern (1988), gerando o índice de laços com membros externos ao grupo e internos no grupo (E-I Index), que permitiu avaliar quanto das relações acontecem entre atores pertencentes ao mesmo grupo e quanto das relações ocorreram entre membros de grupos distintos.

Uma vez realizada a análise estrutural da rede, realizou-se a análise do período com algumas ponderações relacionais, estas avaliadas por meio das medidas egocêntricas. $\mathrm{O}$ objetivo de se extraírem tais medidas foi avaliar se os atores centrais da rede são aqueles com maior poder, ou com melhor acesso ao fluxo de conhecimento e informações, pois de acordo com Hanneman e Riddle (2005), o poder do ego é a dependência do alterego. Para tal, a centralidade é a medida mais utilizada. Na presente análise, quatro medidas de centralidade foram extraídas: centralidade de grau (degree), centralidade de poder (Bonacich), centralidade de proximidade (closeness Eigenvector) e centralidade de intermediação (betweenness) (Hanneman \& Riddle, 2005). Para avaliar a consistência dos achados, realizou-se procedimento adotado por Rossoni (2006), que pretendeu analisar o quanto essas medidas de centralidade para todos os atores seriam correlacionadas. 


\section{A fase embrionária da rede de inovação: O período entre 1999 a 2007}

A formação da rede se deu no ano de 1999, quando da fundação do Instituto de Biologia Molecular do Paraná (IBMP), estabelecendo a partir daquele momento uma parceria entre o Governo do Paraná por meio da SETI (Secretaria da Ciência, Tecnologia e Ensino Superior) e do Tecpar (Instituto de Tecnologia do Paraná), e a Fiocruz (Fundação Oswaldo Cruz). Assim, a ligação entre o IBMP e Fiocruz, traduzida em relações de projetos conjuntos nesse primeiro período de existência da organização, ficou evidenciada pela representação da força dos laços entre essas organizações (Figura 2).

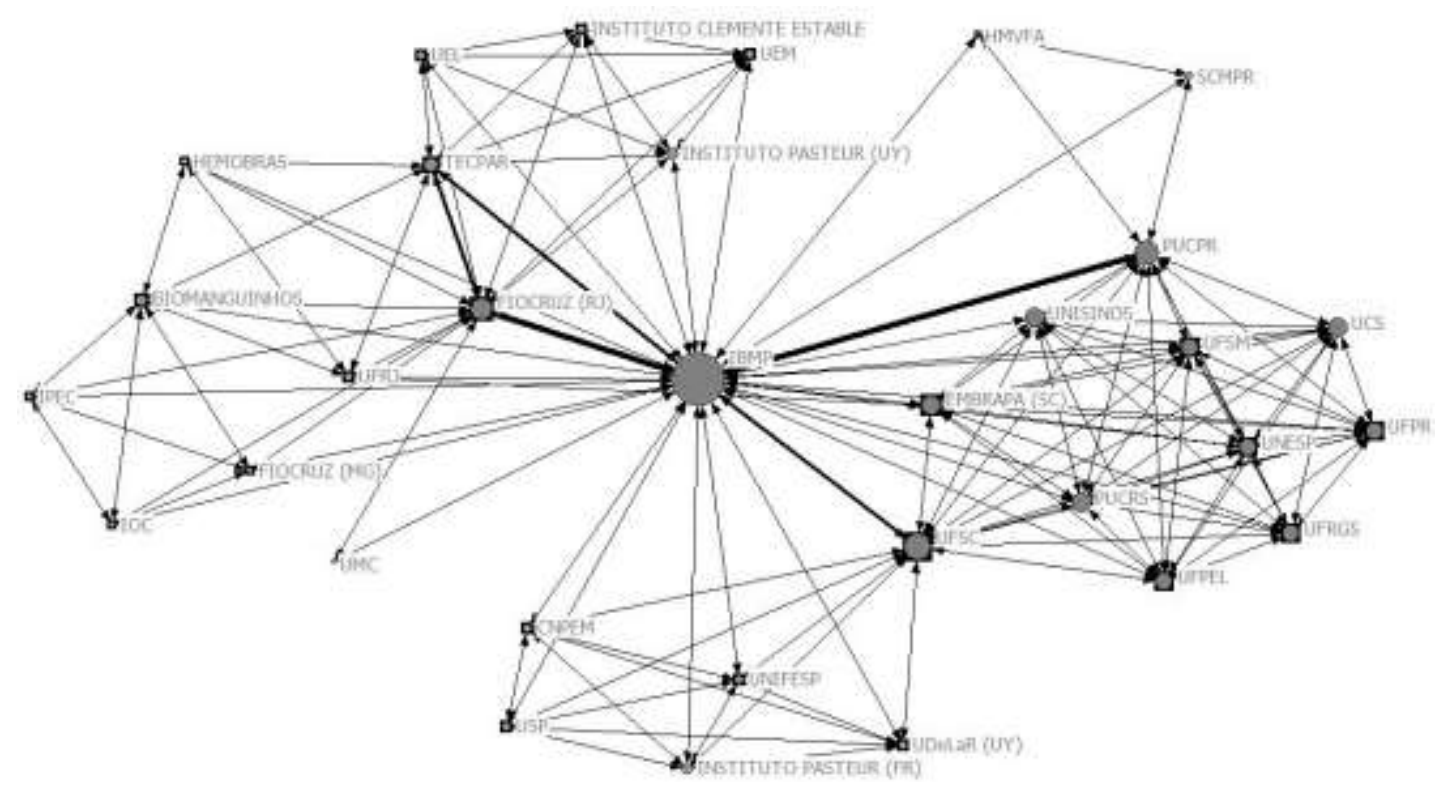

Figura 2. Rede centro-periferia da rede formada pelo IBMP no período de 1999 a 2007

$\mathrm{O}$ ator central da rede era o IBMP, de modo que ele era uma ponte de ligação entre as diversas redes menores dispostas no sociograma. Vale nota que, conforme apresentaram estudos anteriores (Bonfim, Gonçalves, Moreira, \& Jacometti, 2016; Krieger, Souza, Alvarez, Ferreira, \& Moreira, 2013), a área de Biologia Molecular no Paraná era pouco estruturada, praticamente inexistente antes da criação do IBMP. Tal fato ocasionou que as iniciativas voltadas para essa área, em específico, ocorridas no Paraná no período estudado passassem pelo IBMP.

Destaca-se que a rede originária possuía densidade relativamente alta $(0,294$ para $n=31)$ para os moldes de ambientes dinâmicos como o da biotecnologia, caracterizados pela exploração do tipo exploration (Gilsing \& Nooteboom, 2005; Rowley et al., 2000). Contudo, a 
alta densidade se justificou pelo fato de o arranjo estar em formação, aliado ao fato de a área de biotecnologia no Paraná estar em forma embrionária.

Ademais, as medidas extraídas apontaram que o coeficiente de agrupamento (clustering coeficient) geral da rede era muito elevado (coeficiente ponderado de 0,664 e não ponderado de 0,903$)$, o que significa que todos os atores eram cercados por atores com alto índice de agrupamento, apontando para o fechamento da rede. Isto demonstra que os atores estavam ligados em média a 90,3\% dos laços disponíveis na sua vizinhança. O IBMP, sendo o ator mais central da rede apresentou um coeficiente de agrupamento $(0,232)$ mais próximo da densidade geral da rede, sendo a Fiocruz do Rio de Janeiro o segundo ator com menor coeficiente de agrupamento $(0,436)$.

A correlação realizada para verificar a consistência das medidas de agrupamento se mostrou altamente significativa $(p<0,05)$ na ordem de $-0,877$, significando que os dados são consistentes com os padrões esperados de acordo com a teoria dos buracos estruturais (Burt, 1992). Portanto, mesmo a rede apresentando alta densidade, ela apresentou atores com menor grau de agrupamento e consequentemente com alto índice de tamanho efetivo (em especial IBMP e Fiocruz do Rio), garantindo assim o fluxo de informações externas para dentro da rede, por serem buracos estruturais da mesma.

Ao verificar os laços que formavam o ponto de maior vulnerabilidade da rede, a partição resultou em dez subgrupos Lambda ( $\lambda$ ) hierárquicos, sendo que o conjunto Lambda menos vulnerável era formado pelo IBMP e pela UFSC $(\lambda=16)$, seguido pelo subgrupo formado pelo IBMP, UFSC, Fiocruz-RJ e PUC-PR ( $\lambda=13)$, e assim sucessivamente. Esse panorama pode ser visualizado no sociograma da rede do período (Figura 1), notadamente representado pelos laços fortes entre as organizações componentes do conjunto Lambda.

Para compreender o padrão das relações apresentadas, utilizou-se a análise dos índices de laços com membros externos ao grupo e internos no grupo (E-I Index) no que se referiu aos parâmetros adotados como controle (origem, caráter, finalidade e endogenia). Quanto à origem, o índice apontou preponderância dos laços internos sobre os laços externos $(E-I=-0,698)$, ou seja, havia uma grande tendência de fechamento da rede. Tal fator pode ter se dado pois a maioria dos componentes eram oriundos do Brasil. No que se referem aos membros de outros países, no Instituto Pasteur da França todos seus laços eram externos $(E-I=1,000)$. Já os demais membros, oriundos do Uruguai (Instituto Pasteur, Instituto Clemente Estable e Universidad de La República), apresentaram um E-I Index médio de 0,778, o que apontou para forte tendência 
às relações externas, visto que nessa fase da rede, a disponibilidade de laços internos para essas organizações estrangeiras era altamente limitada.

Quanto à finalidade, percebeu-se um equilíbrio na relação entre laços internos e externos $(E-I=0,065)$ com tendência de abertura, de modo que 46,2\% das relações davam-se intragrupos e 53,2\% dessas relações ocorriam com membros externos. A densidade dos laços internos dos grupos $(0,387)$ foi maior do que a densidade geral da rede $(0,294)$, já a densidade dos laços externos foi de $22,6 \%$, menor do que a densidade geral. $\mathrm{O}$ único grupo que apresentou índice negativo, ou seja, era mais propenso às relações internas e ao fechamento do grupo, foi o das universidades $(E-I=-0,342)$. Já o grupo que apresentou maior tendência de ter laços externos, e consequentemente maior índice $E-I(0,727)$, foi o de laboratórios.

No que tange ao caráter da organização, houve um equilíbrio nas relações com membros internos e externos $(E-I=-0,079)$, porém com leve tendência de fechamento. Os dados demonstraram que as organizações públicas tinham maior tendência de colaborar entre si do que com organizações privadas $(E-I=-0,304)$, ao passo que as organizações privadas tendiam a possuir mais laços com organizações públicas do que com seus pares privados $(E-I=0,362)$. Pondera-se que nenhuma organização pública apresentou índice individual positivo, indicando prevalência de laços externos, da mesma maneira que nenhuma organização privada apresentou índice negativo, apontando preferência pelos laços externos, o que pode ter determinado o equilíbrio do E-I Index geral do atributo.

Os dados da pesquisa também apontaram um índice de endogenia relativamente baixo $(E-I=0,104)$, de modo que a tendência no período foi na direção de a Fiocruz manter mais relações com membros externos do que entre suas próprias unidades. O IBMP teve um índice elevado e positivo ( $E-I=0,677)$, o que indica que a maioria dos seus laços ocorreram com organizações que não pertenciam ao Sistema Fiocruz. A Fiocruz $(\mathrm{RJ})$ demonstrou tendência de abertura $(E-I=0,231)$, ao passo que Bio-Manguinhos apresentou tendência para o fechamento de suas relações no âmbito da Fiocruz $(E-I=-0,250)$.

Considerando o fato de o IBMP ser a organização focal do estudo, o instituto apresentou ao menos um laço com todas as organizações (Degree $=31$ ), destacando-se também nos valores referentes ao poder de Bonacich $(4029,35)$ e de intermediação (Between=289,5). O coeficiente de centralidade de proximidade ainda era o maior da rede (Eigenvector $=0,370$ ), porém a discrepância em relação aos outros atores foi menor, ou seja, a distância geodésica entre o IBMP e os outros atores mais centrais do que dos periféricos (os componentes da GeneSul, ao lado de PUC-PR e UFPR) era pequena. 
Outro ponto interessante de se observar foi que a Fiocruz-RJ possuía um baixo índice de centralidade de proximidade (Eigenvector $=0,097$ ), o que significa que sua distância para outros atores centrais da rede era grande. Contudo, a mesma poderia ser considerada, além do IBMP e ao lado da UFSC (Between=25), o maior broker da rede devido ao seu alto grau de centralidade de intermediação (Between=18,5), controlando o fluxo de informações, assim como o acesso de atores periféricos ao centro da rede.

Os resultados apontaram correlação significativa positiva entre todas as variáveis $(p<0,05)$, o que indica que quanto maior a centralidade de grau de um ator, maior o coeficiente de centralidade de poder $(r=0,790)$ e ligação com outros atores centrais da rede, maior o coeficiente de centralidade de proximidade (na ordem de 0,819 ) e maior a centralidade de intermediação $(r=0,803)$. O resultado da correlação foi muito próximo ao obtido por Rossoni (2006), porém diferindo na correlação entre centralidades de grau e intermediação. O autor obteve uma correlação bem inferior à encontrada no presente estudo, o que pode significar que os atores centrais da rede formada pelo IBMP tendiam a colaborar mais com atores periféricos da rede do que entre si, possibilitando a esses atores o acesso ao conhecimento e às informações disponíveis na rede.

\section{A fase de afirmação da rede de inovação: o período entre 2008 e 2014}

Para verificar a dinâmica e evolução da rede, optou-se por realizar o recorte temporal por meio de dois eventos relevantes que aconteceram no mesmo ano: a criação do ICC e a formação da rede INDI-Saúde. A análise compreendeu os dados do período entre os anos de 2008 e 2014 com o objetivo de verificar qual era a posição do arranjo Fiocruz Paraná em um nível de análise mais amplo, ou seja, se realmente a Fiocruz Paraná permaneceu como um hub da rede local.

Seguindo a mesma lógica até aqui empregada, serão apresentados o sociograma (Figura 3) e os dados de coesão estrutural da rede no período. A análise gráfica permite perceber uma mudança dos atores de maior destaque na rede. Um bloco específico se destacou, formado pelo IBMP, pelo ICC (que não existia na rede anterior, porém que foi criado com status de Unidade da Fiocruz) e a UFPR, de modo que a espessura das linhas que representam as relações demonstrou a presença de laços fortes entre esses três membros, formando uma tríade bem definida no grafo. 


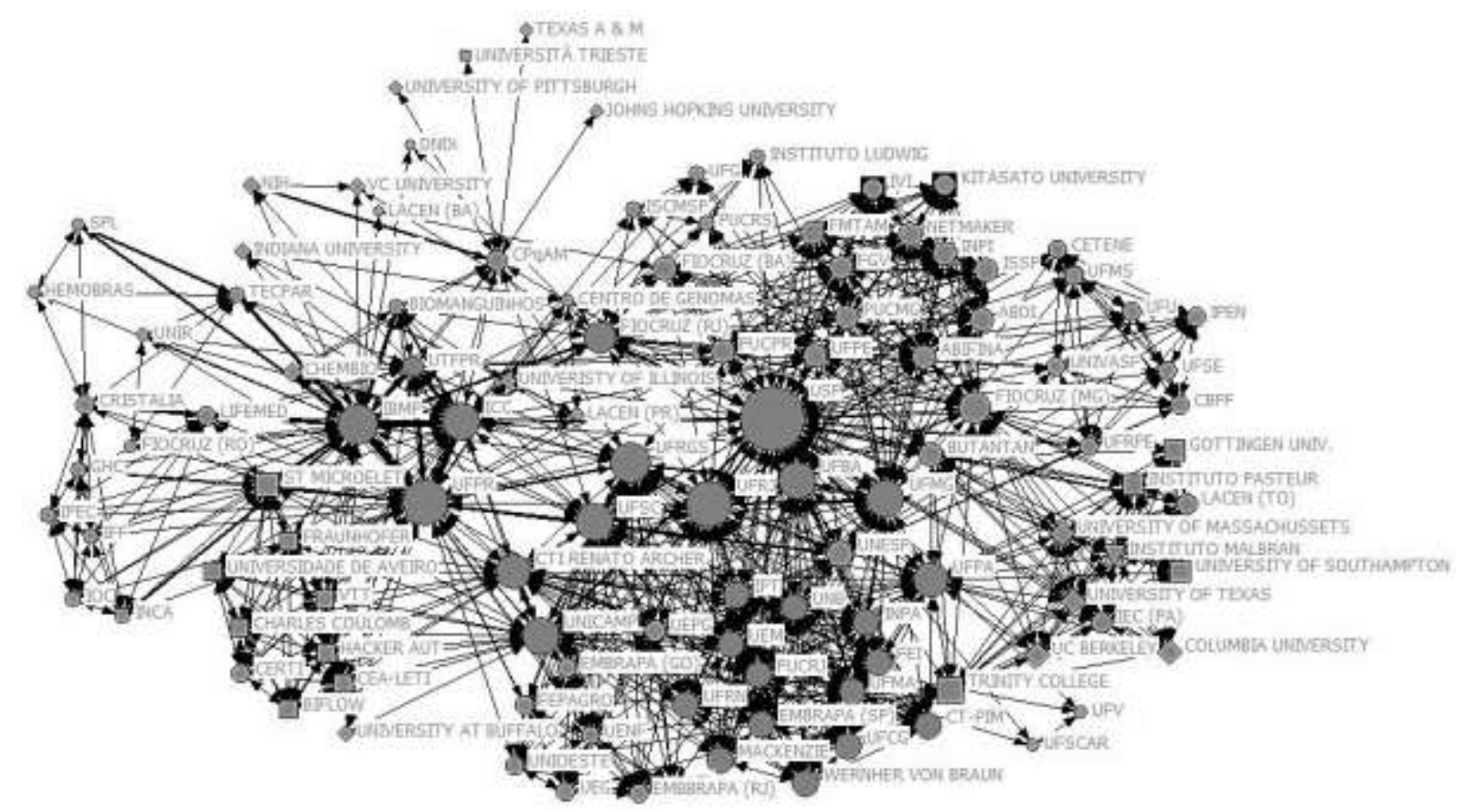

Figura 3. Sociograma da rede centro-periferia do arranjo Fiocruz Paraná no período de 2008 a 2014

Conforme a rede evoluiu, aumentando seu escopo, ela se tornou mais esparsa. Assim, a densidade observada apresentou grande queda, apontando índice de 14,0\%. A tendência maior de abertura se deu, pois, nessa fase da rede houve a busca da diferenciação do pipeline de inovação do arranjo. Ao começar a atuar em áreas antes não atendidas pelo IBMP, os atores da rede precisaram buscar atores externos à rede pela necessidade de complementaridade das habilidades e competências imposta pelos novos projetos. Ao buscar essas novas relações, observou-se a criação de rede entre redes, não apenas entre organizações. No caso da utilização dos microarranjos líquidos para a ampliação dos testes baseados no conceito de Lab-on-a-Chip e Point-of-Care ${ }^{l}$, houve demanda por conhecimentos multidisciplinares não disponíveis na rede, fazendo com que os atores começassem a buscar atores que atuassem fora do ramo da biologia molecular (Departamento de Física da UFPR e Departamento de Engenharia Elétrica da UTFPR).

Os dados confirmaram a tendência de abertura da rede, já que houve também uma redução no coeficiente de agrupamento, tanto no geral $(0,865)$ quanto no ponderado $(0,619)$. Contudo, salienta-se que a tendência de agrupamento e fechamento da rede nas vizinhanças locais ainda foi muito alta se comparada com a densidade total da rede $(0,140)$. O alto índice

\footnotetext{
${ }^{1}$ Lab-on-a-Chip refere-se ao multiteste (teste multiplex) rápido que pode diagnosticar diversas doenças com apenas um aparelho. O conceito point-of-care refere-se à possibilidade da utilização desse diagnóstico direto no ponto de atendimento ao paciente.
} 
de coeficiente de agrupamento geral pode ser explicado pelas medidas dos egos, visto que a maioria dos egos estava ligada a todos os vizinhos possíveis, formando diversas redes menores dentro da rede principal. As organizações que apresentaram coeficiente de agrupamento mais próximo ao índice de densidade geral da rede foram $\operatorname{USP}(0,223), \operatorname{UFPR}(0,241), \operatorname{IBMP}(0,260)$ e $\operatorname{ICC}(0,286)$.

A correlação entre os coeficientes de agrupamento dos egos e o tamanho efetivo da rede se manteve significativa no segundo período estudado $(r=-0,868, p<0,05)$. A manutenção da alta correlação, aliada à queda no índice de densidade geral da rede, apontou para um fluxo ainda maior da informação e dos conhecimentos entre os membros da rede à medida que ela possuía maior abertura. Assim, a alta densidade dos laços dentro das vizinhanças (coeficiente de agrupamento ainda próximo a $80 \%$ ) permitiu maior confiança por meio da força dos laços fortes, favorecendo a exploração por meio da exploitation, indo ao encontro dos argumentos de Coleman (1988). Do mesmo modo, a presença de buracos estruturais na rede que favoreceram o fluxo de informações e a exploração do tipo exploration necessários para a inovação no âmbito multidisciplinar, indo ao encontro das ideias de Burt (1992), tornando o argumento de hibridismo da rede de inovação ainda mais robusto.

Já o procedimento de partição destacou trinta conjuntos Lambda dentro da rede. O conjunto Lambda com maior fator e, portanto, a relação mais importante e menos vulnerável da rede, foi entre a USP e UFPR $(\lambda=47)$, seguido hierarquicamente das relações entre USP, UFPR e UFRJ $(\lambda=45)$ e USP, UFPR, UFRJ, UFRGS E IBMP $(\lambda=38)$.

Outras duas observações foram relevantes nesse momento. A primeira foi que o ICC começou suas atividades no ano de 2009, ou seja, em menos de cinco anos tornou-se um ator importante para a rede $(\lambda=36)$. A segunda foi a presença da ST Microelectronics $(\lambda=21)$ dentre os principais atores no conjunto hierárquico acima apresentado, cujas características eram consideravelmente díspares das que caracterizavam os atores centrais no período anterior por ser uma organização industrial privada de semicondutores, com fins lucrativos e com base no exterior (Itália). A criação de laços da rede da Fiocruz Paraná com a ST Microelectronics por meio da rede PodiTrodi foi fruto da integração do projeto INDI-Saúde com o INCT-Namitec, sendo esse um exemplo clássico de buracos estruturais da rede (Burt, 1992) originados pela necessidade de exploration (Rowley et al., 2000) encontrada pela rede para produzir bionanotecnologia. 
Em seguida, procedeu-se com a verificação dos padrões de relação entre grupos por afiliações ou atributos para o período. No que se refere à origem, o índice $(E-I=-0,548)$ aponta que os grupos tenderam a se relacionar mais entre si do que com membros externos. As organizações brasileiras tenderam a se relacionar entre si $(E-I=-0,759)$, novamente, ponderando que a disponibilidade de organizações nacionais era bem maior do que de estrangeiras. As organizações europeias tiveram E-I Index positivo $(0,223)$, indicando tendência de abertura dentro do grupo, devido, em grande parte, à formação heterogênea do PodiTrodi, o Consórcio Brasil-Europa de diagnósticos de doenças tropicais.

Já as organizações americanas mostraram forte tendência à abertura $(E-I=0,636)$, tendo apenas dezesseis dos seus setenta e dois laços entre si. Em comparação com a rede formada no primeiro período, o grupo formado por origem apresentou tendência de abertura, visto que organizações da Europa, Estados Unidos e Ásia $(E-I=0,882)$ entraram para a rede. No que se refere ao grupo formado por organizações da América Latina, 100\% das relações foram formadas com membros externos ao grupo. A Fiocruz Paraná, em firmando acordo para vigilância epidemiológica de fronteira com Instituto Malbrán (Argentina), Instituto Pasteur e UdeLaR (Uruguai), afetou esse índice no sentido da sua redução ao catalisar a relação entre essas organizações em específico.

No que tange o $E-I$ Index sinalizou maior abertura da rede $(E-I=0,192)$. Ao se observar os índices grupo por grupo, verificou-se que apenas um grupo possuía preponderância das relações com organizações da mesma finalidade, o de universidades. Destaca-se que nesse período entraram para a rede atores de setores antes não representados, como o da indústria, que passou a compor a rede com E-Index $(0,652)$ altamente positivo.

A análise relacionada ao caráter das organizações apresentou E-Index (-0,352) com tendência de fechamento entre os grupos bem maior do que o apresentado no período anterior $(E-I=-0,079)$. As organizações de caráter público apresentaram preponderância dos laços internos $(E-I=-0,581)$, ao passo que as organizações privadas apresentaram preponderância das relações com organizações públicas $(E-I=0,426)$. No âmbito das organizações públicas brasileiras, a Hemobrás $(E-I=0,500)$ foi a única com preponderância das relações com organizações privadas, apresentando maior abertura na sua rede. Os resultados representados pelos E-I Index foram condizentes com os argumentos de Amburgey, Al-Laham, Tzabbar e Aharonson (2008) de que as organizações privadas de biotecnologia frequentemente buscam parcerias com organizações que não visam ao lucro (como institutos de pesquisa e agências governamentais), assim como com laboratórios químicos e farmacêuticos já estabelecidos. 
O exemplo do IBMP ilustra esse argumento, visto que a partir da criação do ICC o mesmo deixou as funções de instituto de pesquisa e de ensino para a Unidade da Fiocruz e buscou se posicionar como uma biotech. O E-I Index do IBMP no período foi relativamente elevado $(0,421)$, demonstrando que a organização buscou organizações públicas para estabelecer relações, contudo, a criação de laços com grandes laboratórios já estabelecidos também foi observada (Chembio na transferência de tecnologia para desenvolvimento dos testes multiplex DDP® e Lifemed na transferência de tecnologia e produção dos testes lab-on-a-chip).

A análise da endogenia das relações entre Unidades da Fiocruz apresentou uma mudança significativa em direção à abertura da rede $(E-I=0,600)$. Neste período, nenhuma unidade da Fiocruz apresentou preponderância das relações com membros internos. Bio-Maguinhos, que possuía índice negativo no período anterior, passou a apresentar um E-I Index de 0,111 positivo, ao passo que o ICC, que não estava presente no período anterior, apresentou elevado índice de abertura mesmo com o IBMP sendo considerado como parte da Fiocruz para efeitos de análise.

Outro parâmetro adotado para comparação entre as redes foi a centralidade dos egos, ou seja, se os atores que possuíam posições privilegiadas na rede no período anterior continuaram sendo os mais poderosos. Os valores apresentados indicaram que os atores que formavam o core visível da rede no grafo eram os atores com maiores índices de centralidade de grau. A USP (Degree $=71)$ apresentou a maior centralidade de grau, sendo o ator com o maior número de relações dentro da rede, possuindo uma diferença significativa para os atores centrais com maior centralidade após a mesma, UFPR (Degree=45) e UFRJ (Degree=45). A UFPR, que possuía um dos maiores índices de centralidade da rede primitiva (Degree=11), e a UFRJ, que possuía baixo índice de centralidade de grau no período anterior (Degree=6), apresentaram um salto de relevância na rede entre os períodos analisados.

Deste modo, o IBMP (Degree $=38)$ deixou de ser o ator central da rede global e de possuir ligação com todos os nós como no período anterior, refletindo a redução da diferença entre as suas medidas de centralidade de grau e as medidas de seus pares. Os dois atores mais centrais da rede entre 1999-2007 ao lado do IBMP, PUC-PR (Degree=20) e UFSC (Degree=37), se tornaram atores menos centrais assim como o próprio IBMP. Outra observação foi de que UFPR e IBMP, ao lado do ICC (Degree=36), apresentaram centralidade na rede periférica formada em torno de si.

No que se refere à centralidade de intermediação, a USP manteve sua posição como ator mais central (Betweenness=1992,740). A UFSC, que no período anterior possuía o status de 
segundo maior broker da rede teve seu status reduzido no período (Betweenness=212,777), e o mesmo fenômeno pôde ser observado com a PUC-PR (Betweenness=55,428). UFPR (Betweennes=903,143) e IBMP (Betweenness=786,798) foram os maiores brokers da rede após a USP, e O ICC, que foi criado efetivamente em 2009, passou a ser um ator altamente intermediador (Betweenness=458,664), ao lado do CPqAM (Betweenness=460,059).

Ao se fazer a análise do índice Eigenvector de centralidade percebeu-se que a USP, mesmo mantendo vantagem no índice de vantagem posicional em relação aos demais (Eigenvector=0,274), apresentou índice mais próximo aos seus pares mais centrais, UFRGS (Eigenvector $=0,203)$, UFMG (Eigenvector $=0,213$ ) e UFRJ (Eigenvector=0,238), significando que esses atores possuíam menor distância geodésica, e, consequentemente, mais proximidade aos atores mais relevantes da rede, o que pode garantir vantagem posicional no acesso aos recursos, conhecimentos e informações disponíveis na rede. O IBMP (Eigenvector=0,096) e o ICC (Eigenvector $=0,097$ ) possuíram desvantagem nesse critério de centralidade, sendo, deste modo, muito distantes do centro da rede.

A última medida de centralidade mensurada, a centralidade de poder, indicou que a USP (Bonacich=10506,634) foi a organização com maior número de laços com outras organizações centrais da rede, seguido pela UFRJ (Bonacich=9111,874). IBMP, ator com maior centralidade de poder no período anterior, teve seu poder reduzido nesse segundo momento (Bonacich=3659,780), ao passo que a UFSC (Bonacich=7616,101) se manteve entre os mais poderosos da rede. Ao que tange o ICC, o mesmo se estabeleceu em uma posição intermediária em relação aos demais atores da rede (Bonacich=3762,767), possuindo índice superior inclusive ao IBMP, membro mais poderoso do período anterior e hierarquicamente mais periférico no período posterior.

As correlações entre os índices de centralidade diferentes se mostraram significativos $(p<0,05)$, apresentando forte relação entre todas as variáveis. Novamente, baseando-se em Rossoni (2006), a alta correlação entre os índices de centralidade de grau e de poder $(r=0,868)$ apontaram que os atores mais centrais da rede tenderam a colaborar com outros atores centrais. Contudo, a forte correlação entre centralidade de grau e de intermediação $(r=0,729)$, apesar de decrescente entre os períodos, indicou que os mesmos ainda buscaram inserir os atores menos centrais nessas redes de colaboração ao se portarem como brokers, facilitando a oxigenação da rede e, consequentemente, o fluxo de informações e conhecimentos que favoreceram a inovação. 
Ao fazer a análise comparativa entre o primeiro e o segundo período, conforme demonstrado na Tabela 1, é possível verificar que a rede expandiu em tamanho, apresentou redução na sua densidade, porém manteve-se com alto grau de agrupamento. Ademais, o aumento no valor do conjunto Lambda demonstra que a rede se tornou menos vulnerável à dissolução. Por fim, no que se refere às relações entre grupos, foi possível que as organizações que compuseram a rede tendiam a se relacionar mais com organizações da sua origem do que com as organizações de outros países.

\section{Tabela 1}

Indicadores adotados no estudo para verificação da dinâmica da rede de inovação do arranjo Fiocruz Paraná entre os períodos 1999-2007 e 2008-2014.

\begin{tabular}{lll}
\hline & \multicolumn{2}{l}{ Período } \\
\cline { 2 - 3 } Indicadores & $1999-2007$ & $2008-2014$ \\
\hline N observações & 31 & 113 \\
Densidade da rede & 0,294 & 0,140 \\
Coeficiente de agrupamento & 0,903 & 0,865 \\
Correlação Clust.Coeff*Struc.Holes & $-0,877$ & $-0,868$ \\
Conjunto Lambda & 16 & 47 \\
E-I Index (origem) & $-0,698$ & $-0,548$ \\
E-I Index (finalidade) & 0,065 & 0,192 \\
E-I Index (caráter) & $-0,079$ & $-0,352$ \\
E-I Index (endogenia) & 0,104 & 0,600 \\
\hline
\end{tabular}

Contudo, a rede apresentou crescimento no grau de internacionalização, de modo que a expansão geográfica, que era limitada apenas à América do Sul e França, se expandiu para outros continentes (Europa, Ásia e América do Norte). Quanto às relações entre organizações de finalidades diferentes, a rede formada possuiu abertura crescente, possibilitando a entrada de hospitais, laboratórios químicos e farmacêuticos e organizações não governamentais (como o DNDi, Drugs for Neglected Diseases initiative no projeto para diagnóstico de Doenças Negligenciadas). 
O outro critério analisado, o caráter da organização, demonstrou índice de fechamento crescente, de modo que as organizações públicas tenderam a se relacionar mais entre si do que com as organizações privadas. O mesmo não foi observado nas organizações privadas, que apresentaram índice positivo, indicando maior abertura desse grupo no seu padrão de relacionamento com organizações públicas. Já quanto à endogenia, verificou-se que com o passar do tempo, a rede se tornou mais aberta às organizações externas, tendo seu índice de abertura no segundo período bem superior ao primeiro período estudado.

\section{Considerações finais}

Buscando entender não apenas como os laços são formados e desfeitos, mas como os campos são formados e estruturados por meio de tais dinâmicas, o presente artigo teve como objetivo analisar como se deu a formação e a evolução de uma rede de inovação em biologia molecular voltada para os diagnósticos em Saúde Pública no sul do Brasil por meio da fundação do arranjo Fiocruz Paraná. Demonstrou-se que ao longo do tempo, com a expansão da rede, maior era o seu grau de abertura no que se refere à origem (mesmo dada a preponderância de organizações nacionais) e à finalidade. Deste modo, houve o aumento no fluxo de conhecimento entre entidades das diferentes finalidades com diferentes arcabouços metodológicos e de conhecimentos especializados, aumentando, assim, a probabilidade de inovação (Crespo, Suire, \& Vicente, 2016; Owen-Smith \& Powell, 2004).

A presente investigação contribuiu também ao demonstrar que durante sua evolução e dinâmica, a rede estudada apresentou mudanças em características além do seu tamanho (número dos laços). Quanto à sua estrutura, a evolução da rede levou ao aparecimento de buracos estruturais (Burt, 1992), porém continuou com elevado adensamento das relações entre as vizinhanças, favorecendo a confiança e a transferência de conhecimento tácito (Hage, Mote, \& Jordan, 2013). A força dos laços entre os atores mais próximos aumentou, levando à maior a quantidade de projetos conjuntos e à potencialização da confiança mútua entre esses atores, necessários para a ocorrer o compartilhamento de conhecimento especializado (Gausdal, Svare, \& Möllering, 2016). Similarmente, com o passar do tempo a rede se tornou mais estável e menos propensa à dissolução, ou seja, houve um aumento na força da rede como um todo, apesar da redução da densidade da mesma entre um período e outro.

O estudo demonstrou que o fator tempo foi chave no processo de inovação. Para sustentar esse argumento, levou-se em consideração que ao analisar a história da rede, uns laços se formam da mesma forma que outros laços se desfazem (Ahuja et al., 2012), contudo os laços passados ficam latentes e podem ser acessados e reincorporados à rede em qualquer momento do tempo por meio do que Ahuja, Soda e Zaheer (2012) chamaram de conteúdo relacional da 
rede. O conteúdo relacional vai ser maior à medida que a rede vai se estabilizando (mesmo que tenha sido criada por tempo determinado) ao longo do tempo, sendo possível acessar contatos que já não incorporam a rede no caso de uma necessidade específica de conhecimento ou de relações que podem ser realizadas por meio desse contato, o que também pode ser um fator chave no processo de inovação.

O presente estudo, contudo, possui limitações. A primeira limitação foi a análise por meio de uma organização focal (Fiocruz Paraná), o que pode levar a alguns vieses no que concerne à importância relativa da organização para o campo de biologia molecular. Do mesmo modo, a coleta predominantemente realizada por meio de dados documentais pode ter levado à não consideração de laços informais, porém relevantes para a formação, evolução, e dinâmica da rede biotecnológica estudada. Deste modo, para estudos futuros recomenda-se que as dinâmicas da rede sejam investigas longitudinalmente, possibilitando não apenas compreender como a rede evolui, mas também qual o real papel dos laços informais durante este processo.

\section{Referências}

Abosag, I., Yen, D. A., \& Barnes, B. R. (2016). What is dark about the dark-side of business relationships? Industrial Marketing Management, 55, 5-9.

Ahuja, G. (2000). Collaboration Networks, Structural Holes, and Innovation: A Longitudinal Study. Administrative Science Quarterly, 45(3), 425-455.

Ahuja, G., Soda, G., \& Zaheer, A. (2012). The genesis and dynamics of organizational networks. Organization Science, 23(2), 434-448.

Amburgey, T. L., Al-Laham, A., Tzabbar, D., \& Aharonson, B. (2008). The structural evolution of multiplex organizational networks: research and commerce in biotechnology. In J. A. C. Baum \& T. J. Rowley (Eds.), Network Strategy: advances in strategic management (Vol. 25, pp. 171-209). Bingley, UK: Emerald.

Bonfim, L. R. C., Gonçalves, S. A., Moreira, M. S., \& Jacometti, M. (2016). Institutional Entrepreneurship and Improvement of Quality of Life: the formation and legitimation of a public-private innovation network in molecular biology applied to public health in Southern Brazil. In J. C. C. Leitão \& H. M. B. Alves (Eds.), Innovative and entrepreneurial practices in public institutions: a quality-of-life approach (pp. 123-154). Cham: Springer.

Borgatti, S. P., Everett, M. G., \& Freeman, L. C. (2002). Ucinet for Windows: Software for social network analysis.

Borgatti, S. P., Everett, M. G., \& Shirey, P. R. (1990). LS sets, lambda sets and other cohesive subsets. Social networks, 12(4), 337-357.

Burt, R. S. (1992). Structural holes: the social structure of competition. In R. G. Eccles \& N. Nohria (Eds.), Network and organizations: structure, form and action (pp. 57-93). Boston, MA: Harvard Business School Press.

Burt, R. S. (2000). Decay functions. Social networks, 22(1), 1-28. 
Burt, R. S. (2001). Structural holes versus network closure as social capital. In N. Lin, K. S. Cook, \& R. S. Burt (Eds.), Social capital: theory and research (pp. 31-56). New Brunswick, NJ: Transaction.

Castilla, E. J., Hwang, H., Granovetter, E., \& Granovetter, M. S. (2000). Social networks in silicon valley. In C. M. Lee, W. F. Miller, M. G. Hancock, \& H. S. Rowen (Eds.), The Silicon Valley edge: A habitat for innovation and entrepreneurship (pp. 218-247). Stanford, CA: Stanford University Press.

Coleman, J. S. (1988). Social capital in the creation of human capital. American Journal of Sociology, 94(Supplement), S95-S120.

Crespo, J., Suire, R., \& Vicente, J. (2016). Network structural properties for cluster long-run dynamics: evidence from collaborative R\&D networks in the European mobile phone industry. Industrial and Corporate Change, 25(2), 261-282.

Emirbayer, M., \& Goodwin, J. (1994). Network analysis, culture, and the problem of agency. American Journal of Sociology, 99(6), 1411-1454.

Funk, R. J., \& Owen-Smith, J. (2017). A dynamic network measure of technological change. Management Science, 63(3), 791-817.

Gausdal, A. H., Svare, H., \& Möllering, G. (2016). Why don't all high-trust networks achieve strong network benefits? A case-based exploration of cooperation in Norwegian SME networks. Journal of Trust Research, 6(2), 194-212.

Gilsing, V., \& Nooteboom, B. (2005). Density and strength of ties in innovation networks: an analysis of multimedia and biotechnology. European Management Review, 2(3), 179-197.

Grandori, A. (1997). An organizational assessment of interfirm coordination modes. Organization Studies, 18(6), 897-925. doi:10.1177/017084069701800601

Grandori, A., \& Soda, G. (1995). Inter-firm networks: antecedents, mechanisms and forms. Organization Studies, 16(2), 183-214.

Granovetter, M. S. (1973). The Strength of Weak Ties. American Journal of Sociology, 78(6), 1360-1380.

Granovetter, M. S. (1985). Economic action and social structure: the problem of embeddedness. American Journal of Sociology, 91(3), 481-510.

Gulati, R., Nohria, N., \& Zaheer, A. (2000). Strategic networks. Strategic Management Journal, 21(3), 203.

Hage, J., Mote, J. E., \& Jordan, G. B. (2013). Ideas, innovations, and networks: a new policy model based on the evolution of knowledge. Policy Sciences, 46(2), 199-216.

Hanneman, R. A., \& Riddle, M. (2005). Introduction to social network methods. Riverside, CA: University of California Riverside.

Krackhardt, D. (1992). The strength of strong ties: the importance of philos in organizations. In N. Nohria \& R. G. Eccles (Eds.), Networks and Organizations: Structure, Form, and Action (pp. 216-239). Boston, MA: Harvard Business School Press.

Krackhardt, D., \& Stern, R. N. (1988). Informal networks and organizational crises: An experimental simulation. Social Psychology Quarterly, 51(2), 123-140.

Krieger, M. A., Souza, R., Alvarez, P., Ferreira, A., \& Moreira, M. S. (2013). Cooperação técnico-científica entre instituições públicas e privadas para incorporação tecnológica: impacto da nacionalização do teste NAT HIV e HCV na ampliação do acesso e na formação de base tecnológica no âmbito do Sistema Único de Saúde. Divulgação para Saúde em Debate, 50, 102-112.

Lavie, D. (2006). The competitive advantage of interconnected firms: An extension of the resource-based view. Academy of Management Review, 31(3), 638-658.

Lee, S., \& Monge, P. (2011). The coevolution of multiplex communication networks in organizational communities. Journal of Communication, 61(4), 758-779. 
Owen-Smith, J., \& Powell, W. W. (2004). Knowledge Networks as Channels and Conduits: The Effects of Spillovers in the Boston Biotechnology Community. Organization Science, 15(1), 5-21.

Phelps, C. C. (2010). A longitudinal study of the influence of alliance network structure and composition on firm exploratory innovation. Academy of Management Journal, 53(4), 890913.

Powell, W. W. (1990). Neither market nor hierarchy: network forms of organization. Research in Organizational Behavior, 12, 295-336.

Powell, W. W., Koput, K. W., \& Smith-Doerr, L. (1996). Interorganizational collaboration and the locus of innovation: Networks of learning in biotechnology. Administrative Science Quarterly, 41(1), 116-145.

Powell, W. W., White, D. R., Koput, K. W., \& Owen-Smith, J. (2005). Network dynamics and field evolution: The growth of interorganizational collaboration in the life sciences. American Journal of Sociology, 110(4), 1132-1205. doi:10.1086/421508

Rossoni, L. (2006). A dinâmica das relações no campo da pesquisa em organizações $e$ estratégia no Brasil: uma análise institucional. (Mestrado em Administração), Universidade Federal do Paraná, Curitiba, PR.

Rowley, T., Behrens, D., \& Krackhardt, D. (2000). Redundant governance structures: An analysis of structural and relational embeddedness in the steel and semiconductor industries. Strategic Management Journal, 21(3), 369-386.

Soda, G., Usai, A., \& Zaheer, A. (2004). Network memory: The influence of past and current networks on performance. Academy of Management Journal, 47(6), 893-906.

Thorelli, H. B. (1986). Networks: between markets and hierarchies. Strategic Management Journal, 7(1), 37-51.

Vasudeva, G., Zaheer, A., \& Hernandez, E. (2013). The Embeddedness of Networks: Institutions, Structural Holes, and Innovativeness in the Fuel Cell Industry. Organization Science, 24(3), 645-663.

Williamson, O. E. (1991). Comparative economic organization: the analysis of discrete structural alternatives. Administrative Science Quarterly, 36(2), 269-296.

Wood Jr., T. (2010). Organizações híbridas. Revista de Administração de Empresas, 50(2), 241-

247. 